\title{
Emotional Impact on the User Experience in the Virtual Learning Environment
}

\author{
Tiago do Carmo Nogueira ${ }^{1}$, Raimundo Nonato de Araujo Soares Neto ${ }^{2}$ \\ ${ }^{1}$ Departamento de Informática \\ Instituto Federal de Educação, Ciência e Tecnologia de Mato Grosso (IFMT) \\ Estrada de acesso a BR-158, Radial José Maurício Zampa, s/n - CEP: 78600-000 \\ ${ }^{2}$ Centro de Ensino e Aprendizagem em Rede (CEAR) \\ Universidade Estadual de Goiás (UEG) \\ Brasil Sul, No 2800 - Jardim Gonçalves - CEP: 75123-315 \\ tiago.nogueira@bag.ifmt.edu.br, raimundonsnetolgmail.com
}

\begin{abstract}
This article aims to analyze the emotional impact on the user experience $(U X)$ during interactions performed in the virtual learning environment. In orther to do this, it was applied the Affect Grid that classifies eight emotions experienced by users. The work included the participation of 12 students of the course of the distance Degree in Computing at the State University of Goiás. It was analyzed and classified 60 responses during the interactions of the tasks performed in the virtual learning environment, observing the emotional impact of each task. The results suggest, that through the application of methods UX is possible to extract the emotions felt by the students during interactions in virtual learning environments.
\end{abstract}

Resumo. Este artigo analisou o Impacto Emocional da Experiência dos Usuários $(U X)$ durante as interações realizadas em Ambientes Virtuais de Aprendizagens. Para tal, aplicou-se o método Affect Grid que classifica oito tipos de emoções sentidas pelos usuários. O trabalho contou com uma amostra de 12 alunos do curso de Licenciatura em Computação da Universidade Estadual de Goiás. Foram analisadas e classificadas sessenta respostas durante as interações das tarefas executadas no Ambiente Virtual de Aprendizagem, observando o Impacto Emocional de cada tarefa. Os resultados surgeriram que, através da aplicação de métodos $U X$ é possível extrair as emoções sentidas pelos usuários durante as interações em Ambientes Virtuais de Aprendizagem, contribuindo significativamente para a qualidade na Usabilidade nestes ambientes.

\section{Introdução}

Desde a segunda metade do século XX, diversas transformações têm ocorrido nos processos de ensino- aprendizagem, proporcionadas em grande parte pelo crescente avanço das tecnologias [Pereira et al. 2007]. Tais mudanças integram um contexto no qual os indivíduos buscam por capacitação em um mundo cada vez mais globalizado, visando, dessa forma a sua própria inserção no competitivo mercado de trabalho.

Nota-se uma alteração no paradigma educacional, visto que, antes, a demanda era formada exclusivamente por alunos que frequentavam salas de aulas físicas de escolas 
VI Congresso Brasileiro de Informática na Educação (CBIE 2017)

Anais do XXIII Workshop de Informática na Escola (WIE 2017)

e de universidades. Atualmente, por meio da utilização de tecnologias da informação e comunicação (TICs), essas ofertas de cursos passaram também a ser disponibilizadas por outras plataformas que não necessitam de um ambiente físico para o compartilhamento do conhecimento.

Analisando os últimos anos, é possível observar a inserção e a massificação do uso de Ambientes Virtuais de Aprendizagem (AVA) na forma de instrumento de apoio educacional, tanto nas instituições privadas quanto nas públicas; auxiliando no ensino, na pesquisa e na extensão. Os AVAs consistem em mídias que utilizam o ciberespaço para transmitir o conteúdo informacional e educacional; permitindo, assim, as interações entre os atores no processo ensino-aprendizagem [Pereira et al. 2007]. Nessa perspectiva, com o aumento de cursos na modalidade a distância (EaD), configurou-se um cenário de desafios para professores, alunos e tutores em relação ao uso dessas ferramentas computacionais.

Segundo dados apresentados pela Universidade Aberta do Brasil (UAB), foram ofertados 912 cursos na modalidade $\mathrm{EaD}$ em 2014. A região Centro-Oeste, por exemplo, possui 143 cursos, sendo 53 cursos de nivel superior, 59 cursos de pós-graduacão e 31 cursos de aperfeiçoamento e extensão [Alves et al. 2015].

De acordo com o Instituto Nacional de Estudos e Pesquisas Educacionais Anísio Teixeira (INEP), entre 2012 e 2013 as matrículas em cursos EaD já representavam cerca de $15 \%$ do total de matrículas em cursos superiores [Ferreira and Elia 2013]. No Estado de Goiás, em 2014 os cursos a distância cresceram 54\% em relação a 2013. Na rede privada cresceu 54\%, isto é; 15 mil alunos em 2013 para 23 mil em 2014. Na rede pública, o índice aumentou em 51\% , ou seja; 458 alunos em 2013 para 692 em 2014 [Semesp 2016]. Este aumento de matrículas também levou ao surgimento de novos desafios; entre eles, o controle da evasão dos alunos nos cursos ofertados por esta modalidade.

Atualmente, uns dos maiores desafios do ensino a distância é controlar o alto índice de evasão [Ferreira and Elia 2013]. Entre os problemas que contribuem para o aumento desse índice, destaca-se a falta de usabilidade em AVAs; ocasionando assim, a desistência por parte do aluno em utilizá-la. Diante disso, existe a necessidade de se investigar os atributos pragmáticos da experiência do usuários (do inglês, User eXperience - UX) em ambientes virtuais de aprendizagem, verificando o impacto experiencial dessas ferramentas nos cursos EaD.

Este artigo apresenta os resultados das análises do impacto emocional da UX durante as interações utilizando o AVA do curso de Licenciatura em Computação da Universidade Estadual de Goiás (UEG). Esta análise conclui que por meio da aplicação dos métodos de UX, foi possível extrair as emoções sentidas pelos alunos durante as interações; classificando-as em prazerosas e desprazerosas, sonolentas e animadas, depressivas e estressantes, relaxantes e excitantes.

O artigo está organizado da seguinte forma: a Seção 2 apresenta os trabalhos relacionados e a Seção 3 apresenta uma breve fundamentação teórica sobre UX. A Seção 4 descreve a metodologia utilizada no trabalho e a Seção 5 analisa e discute os principais resultados encontrados. Por fim, na Seção 6 são apresentadas as conclusões e perspectivas de trabalhos futuros. 
VI Congresso Brasileiro de Informática na Educação (CBIE 2017)

Anais do XXIII Workshop de Informática na Escola (WIE 2017)

\section{Trabalhos Relacionados}

Nesta última década, vários trabalhos foram propostos visando mensurar a experiência emocional em AVAs, especificamente sob aspectos afetivos nas interações com a ferramenta Moodle. Segundo Longhi et al. (2010), para mensurar estes aspectos pode ser realizado um mapeamento do estado do ânimo do aluno durante estas interações. Os autores utilizam a Roda dos Estados Afetivo (REA) para mensurar atributos emocionais dos usuários, identificando atributos de satisfação e insatisfação; e de desânimo e ânimo, definidos a partir de grupos emocionais [Longhi et al. 2010].

Por meio da aplicação do instrumento REA, pode-se analisar a disposição afetiva do aluno em um AVA, identificando-se 20 (vinte) famílias afetivas em 04 (quatro) quadrantes, as quais podem ser utilizadas para o desenvolvimento de regras comportamentais aplicando-se a mineração de dados em AVA [Santos and Bercht 2014].

Para mensurar essas emoções, pode-se utilizar teorias da semântica lexical computacional, identificando sentimentos como apoio à atividade docente em AVAs. Esta abordagem pode obter resultados com ganhos significativos em relação às demais abordagens existentes [Rigo et al. 2013].

Ademais, outras propostas apresentam a modelagem e desenvolvimento de agentes pedagógicos [Frozza et al. 2009] e gamificação [Rezende et al. 2013] para estimular as emoções relacionadas à aquisição de conhecimento; auxiliando, assim, no processo ensino-aprendizagem em ambientes colaborativos.

Em outros trabalhos são analisados fatores que influenciam no desempenho educacional dos alunos. Por exemplo, a identificação de variáveis que possuem uma relação direta com o desempenho de alunos iniciantes em cursos ofertados na modalidade a distância [Ramos et al. 2014]. Pode-se, inclusive, aplicar questionários no início do curso, cruzando algumas variáveis com o desempenho de cada aluno.

Portanto, há necessidade de se investigar as percepções emocionais sentidas pelos alunos durante a oferta de disciplinas a distância, visando mensurar o impacto emocional na experiência do usuários (UX) durante as interações com o Moodle. Na Seção 3 são apresentados o conceito e o instrumento de UX utilizados neste trabalho para mensurar essas emoções sentidas durante as interações em um AVA.

\section{Design da Experiência do Usuário}

O Design da Experiência do Usuário (do inglês, Designing for User Experiences - DUX) pode ser caracterizado pela criação e sincronização dos elementos que afetam a experiência do usuário em websites, e; em particular, nos processos de interação em Ambientes Virtuais de Aprendizagem. Neste caso, analisa-se o comportamento dos alunos durante as interações no processo de ensino e aprendizagem dos cursos a distância.

Ao contrário da Usabilidade, o DUX pode ter um conjunto mais subjetivo de atributos que mensuram as percepções dos usuários nas interações em AVA [Sutcliffe and Hart 2013]. Nesse sentido, a UX é um instrumento importante para alcançar os objetivos das aplicações Web, possibilitando avaliar os aspectos subjetivos dos efeitos parciais ou totais nas interações em AVA [Hartson and Pyla 2012]. O impacto emocional, nesse contexto, se torna um atributo importante para a qualidade experiencial 
VI Congresso Brasileiro de Informática na Educação (CBIE 2017)

Anais do XXIII Workshop de Informática na Escola (WIE 2017)

dos alunos durante as realizações das tarefas propostas pelas disciplinas disponibilizadas em AVA.

Além do impacto emocional, os aspectos de UX são compostos por atributos de usabilidade, funcionalidade e utilidade. Os atributos de usabilidade abordam os aspectos da eficiência e eficácia das aplicações, produtividade e facilidade de uso, capacidade de aprendizagem e retenção [Nielsen 1994]. Nessa perspectiva, o impacto emocional em UX investiga atributos subjetivos de usabilidade, os quais são classificados como de efeito positivo ou de efeito negativo nas interações em AVA [Nogueira et al. 2017].

Para mensurar o impacto emocional dos alunos em AVA, a UX disponibiliza vários métodos consolidados da usabilidade. O Affect Grid é um dos métodos de UX que possibilita classificar os aspectos emocionais sentidos pelos alunos durante as interações [Russell et al. 1989]. Este método é composto por um questionário e uma grid. O questionário utiliza uma pergunta objetiva para identificar as emoções sentidas pelos alunos; por exemplo, como o aluno se sentiu ao realizar a tarefa proposta. Ao responder, o aluno escolhe uma nota em uma escala de 1 a 9 - (desprazer a prazer) - (eixo X) e uma nota, também em uma escala de 1 a 9 - (sonolento a animado) - (eixo Y). Observa-se, por meio desta pergunta, que os alunos possuem o conhecimento de apenas quatros atributos: desprazer, prazer, sonolento e animado. Após a coleta dessa resposta, a interseção entre as notas fornecidas pelo aluno no eixo $\mathrm{X}$ e no eixo $\mathrm{Y}$ são contabilizadas em um espaço na grid. Por exemplo, se um aluno fornecer nota 7 para desprazer-prazer (eixo X) e uma nota 4 para sonolento-animado (eixo Y), a interseção será inserida e contabilizada em um espaço no quarto quadrante da grid. A Figura 1 apresenta a inserção dessa nota no método Affect Grid.

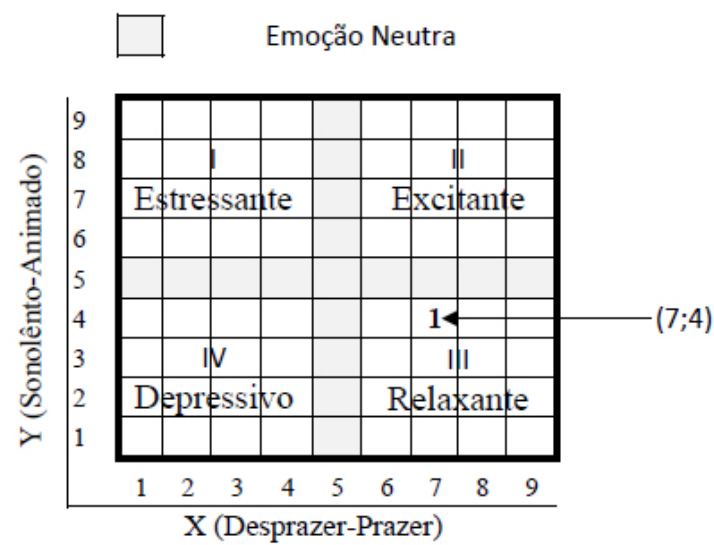

Figura 1. Interseção dos eixos $X$ e $Y$ do Affect Grid.

Após a identificação da interseção entre o eixo horizontal e o vertical na grid, é possível classificar a interação realizada por este aluno, nos demais atributos de UX, classificando, desse modo; a interação como emocionalmente estressante ou depressiva, excitante ou relaxante.

\section{Metodologia}

Buscando uma maneira de avaliar os atributos subjetivos de UX, adotou-se uma abordagem exploratória com questionários de UX, utilizando-se indicadores qualitativos que 
VI Congresso Brasileiro de Informática na Educação (CBIE 2017)

Anais do XXIII Workshop de Informática na Escola (WIE 2017)

auxiliam na compreensão dos fenômenos pragmáticos de UX. Segundo Maule (2012), a abordagem exploratória auxilia na pesquisa com os usuários, investigando o contexto em que são realizadas as tarefas pré-determinadas, ajudando assim a entender os hábitos, motivações e comportamentos dos diferentes tipos de usuários [Maule 2012]. Neste sentido, a metodologia proposta foi dividida em 03 (três) etapas: aplicação das tarefas de interação, aplicação do método Affect Grid (Figura 1) e análise das respostas com extração e classificação dos resultados.

A pesquisa foi realizada entre os meses de Fevereiro a Março de 2015 no AVA do curso de Licenciatura em Computação da Universidade Estadual de Goiás (UEG). Para tal, foi proposta a realização de cinco tarefas durante a disciplina de Fundamentos para o Desenvolvimento de Sistemas. Os alunos necessitavam realizar as seguintes tarefas: fórum de discussão (T1), chat da disciplina (T2), realização de uploads das atividades (T3), troca de mensagens com os colegas de classe (T4) e consulta ao Wiki da disciplina (T5). Com isso, foram analisadas, um total de 60 respostas após a realização das tarefas propostas; avaliando assim, as interações nas principais funcionalidades que compõem o AVA.

Todas as tarefas que foram propostas objetivavam-se apenas em verificar a usabilidade dos recursos disponíveis no AVA. Assim, tais tarefas sugeridas eram fictícias, simulando apenas as ações dos estudantes no AVA. Por exemplo, ao solicitar o envio de um arquivo para a plataforma, não eram necessariamente obrigados à criarem ou redigirem o conteúdo do arquivo, mas apenas enviá-los (arquivo em branco). Neste sentido, testavase apenas a experiência do usuário durante a interação com o mecanismos de upload da plataforma.

\section{Resultados e Discussões}

Nesta seção apresentam-se os resultados e discussões sobre o Impacto Emocional na Experiência dos Usuários em Ambientes Virtuais de Aprendizagem. Para tal, na subseção 5.1 apresenta-se a amostra da pesquisa e na subseção 5.2 os resultados obtidos.

\subsection{Identificação da Amostra}

A amostra da pesquisa contou com 12 (doze) alunos, sendo cerca de 58\% (cinquenta e oito por cento) do sexo masculino e $42 \%$ (quarenta e dois por cento) do sexo feminino, com idade média de 31 anos. 58,3\% (cinquenta e oito vírgula três por cento) dos alunos utilizavam recursos do AVA há mais de 1 ano e menos de 2 anos; 33\% (trinta e três por cento) utilizam recursos do AVA há mais de 2 anos e menos de 4 anos e 8,3\% (oito vírgula três por cento) utilizam há mais de 4 anos.

Em relação à quantidade de horas por semana que os alunos utilizam os recursos do AVA, cerca de $41 \%$ (quarenta e um por cento) o utilizam menos de 2 horas por semana, $25 \%$ (vinte e cinco por cento) o utilizam de 2 a 5 horas e $33,3 \%$ (trinta e três por cento) utilizam de 5 a 10 horas por semana.

Após a realização das tarefas propostas (T1, T2, T3, T4 e T5), o usuário deveria responder à seguinte questão: “Como você se sentiu após a realização dessa interação proposta pela tarefa?". Para mensurar a resposta do usuário, aplicou-se a escala de Likert [Likert 1932], entre 1 e 9, utilizada pelo método Affect Grid [Russell et al. 1989]. 
VI Congresso Brasileiro de Informática na Educação (CBIE 2017)

Anais do XXIII Workshop de Informática na Escola (WIE 2017)

\subsection{Extração dos Resultados}

Após a realização das tarefas e da aplicação do método de UX, foram coletadas 60 respostas resultantes da aplicação do Affect Grid. Cada aluno (sujeito da pesquisa) realizou 5 (cinco) tarefas, respondendo; portanto, 5 (cinco) questionários. O resultado da análise obtida pela aplicação do método na coleta das respostas é apresentada na Tabela 1.

Tabela 1. Quantidade de emoções sentidas pelos alunos durante as tarefas

\begin{tabular}{|l|c|c|c|c|c|c|}
\hline \multirow{2}{*}{ Atributos Emocionais } & \multicolumn{5}{|c|}{ Tarefas } & Total \\
\cline { 2 - 7 } & T1 & T2 & T3 & T4 & T5 & \\
\hline Prazer & 11 & 8 & 3 & 9 & 3 & 35 \\
\hline Desprazer & 0 & 2 & 5 & 3 & 8 & 18 \\
\hline Neutra (PD) & 1 & 2 & 4 & 1 & 0 & 8 \\
\hline Sonolento & 9 & 6 & 3 & 5 & 3 & 26 \\
\hline Animado & 3 & 4 & 4 & 4 & 4 & 19 \\
\hline Neutra (SA) & 0 & 2 & 5 & 3 & 5 & 15 \\
\hline
\end{tabular}

A Tabela 1 apresenta a quantidade de respostas resultante da interseção do eixo $\mathrm{X}$ (Prazer-Desprazer) e do eixo Y (Sonolento-Animado) (veja Figura 1). Pode-se observar que 34 (trinta e quatro) respostas classificaram as tarefas como prazerosas sob o impacto emocional nas interações no AVA, 18 (dezoito) respostas classificaram as tarefas como desprazerosas e 8 (oito) respostas classificaram as tarefas como emocionalmente neutras. No aspecto sonolência e animação, 26 (vinte e seis) respostas classificaram o impacto emocional como sonolento, 19 (dezenove) classificaram como animado e 15 (quinze) respostas classificaram as tarefas como emocionalmente neutras.

Neste sentido, após a análise e aplicação do método, foi possível classificar as respostas em mais quatro atributos emocionais de UX: emoções depressivas ou estressantes, relaxantes ou excitantes. A Figura 2 demostra o percentual das respostas que classificaram as tarefas sob essas emoções.

Após a análise e aplicação do método, cerca de $8 \%$ (oito por cento) das respostas dos alunos identificaram as interações no AVA como depressivas e cerca de $6 \%$ (seis por cento) como estressantes. Cerca de $28 \%$ (vinte e oito por cento) das respostas dos alunos identificaram as interações no AVA como relaxantes e cerca de $18 \%$ (dezoito por cento) como excitante. Observa-se que cerca de $38 \%$ (trinta e oito por cento) das respostas dos alunos identificaram as interações como emocionalmente neutras.

Após essa extração, foi analisado o impacto emocional da experiência do usuário para cada tarefa proposta, com o objetivo de avaliar as diferenças e dificuldades encontradas pelos alunos nas interações do AVA. Nesse aspecto, a tarefa T1 (Fórum de discussão) obteve um resultado de $75 \%$ (setenta e cinco por cento) das respostas dos alunos identificando-a como uma interação emocionalmente relaxante. Além disso, cerca de 16\% (dezesseis por cento) identificaram como uma interação emocionalmente excitante e cerca de $8 \%$ (oito por cento) das respostas foram classificadas como comportamentos emocionais neutros. A Figura 3 apresenta a avaliação do impacto emocional na interação dos usuários nas tarefas T1 (Fórum de discussão), T2 (chat da disciplina), T3 (uploads das atividades), T4 (troca de mensagens) e T5 (consulta ao Wiki). 
VI Congresso Brasileiro de Informática na Educação (CBIE 2017)

Anais do XXIII Workshop de Informática na Escola (WIE 2017)

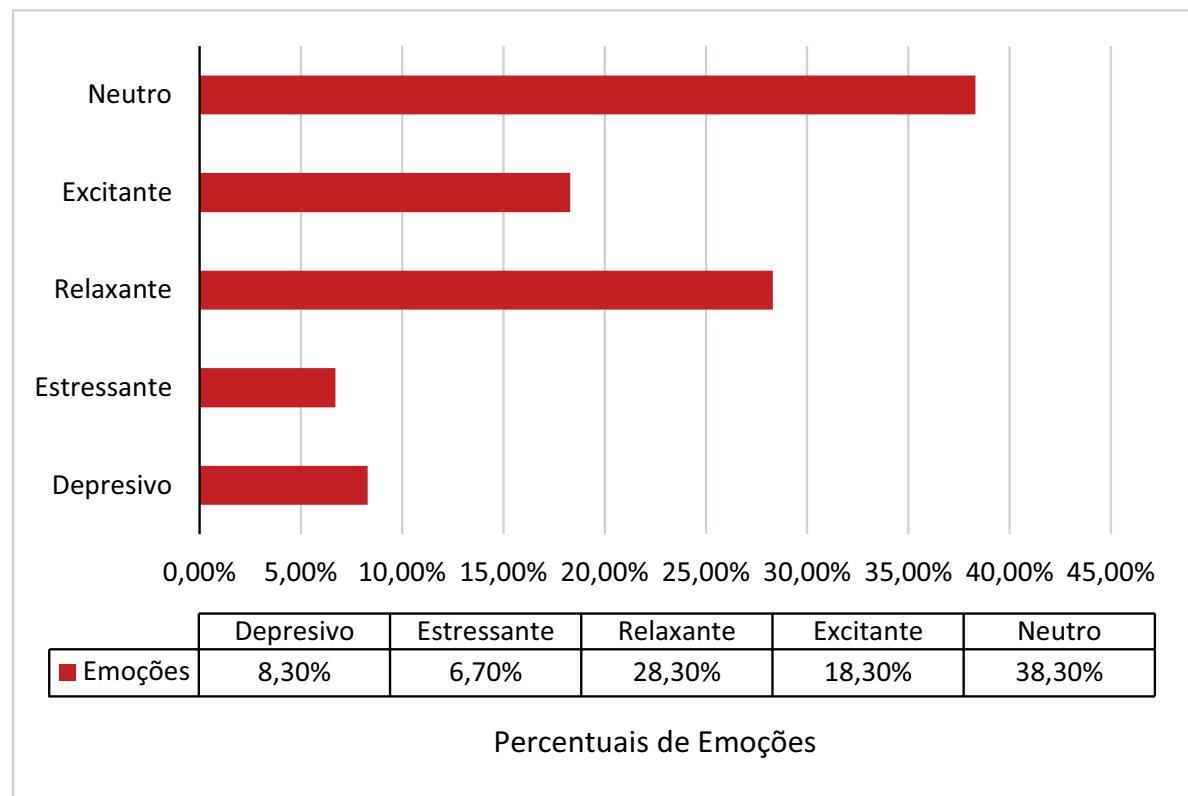

Figura 2. Percentual de respostas identificadas por emoções depressivas, estressantes, relaxantes e excitantes.

Para a tarefa T2 (chat da disciplina) foi obtido um resultado de 16,6\% (dezesseis vírgula seis por cento) das respostas dos alunos, identificando-a como uma interação emocionalmente depressiva. $25 \%$ (vinte e cinco por cento) identificaram como uma interação emocionalmente relaxante. Além disso, $25 \%$ (vinte e cinco por cento) classificaram como uma interação emocionalmente excitante e 33,3\% (trinta e três vírgula três por cento) das respostas foram classificadas como comportamentos emocionais neutros.

A tarefa T3 (uploads das atividades) obteve como resultado 16,7\% (dezesseis vírgula sete por cento) das respostas dos alunos identificando-a como uma interação emocionalmente estressante e $8,3 \%$ (oito vírgula três por cento) identificaram como uma interação emocionalmente depressiva. $75 \%$ (setenta e cinco por cento) das respostas foram classificadas como comportamentos emocionais neutros.

$\mathrm{Na}$ tarefa T4 (troca de mensagens), o resultado foi de 8,3\% (oito vírgula três por cento) das respostas dos alunos identificando-a como uma interação emocionalmente estressante. $41,7 \%$ (quarenta e um vírgula sete por cento) identificaram como uma interação emocionalmente relaxante, $25 \%$ (vinte e cinco por cento) das respostas dos alunos identificaram como uma interação emocionalmente excitante e $25 \%$ (vinte e cinco por cento) das respostas foram classificadas como comportamentos emocionais neutros.

Enfim, a tarefa T5 (consulta ao Wiki) apresentou 16,7\% (dezesseis vírgula sete por cento) das respostas dos alunos identificando-a como uma interação emocionalmente depressiva e estressante respectivamente. $25 \%$ (vinte e cinco por cento) das respostas dos alunos identificando-a como uma interação emocionalmente excitante e 41,7\% (quarenta e um vírgula sete por cento) das respostas foram classificadas como comportamentos emocionais neutros.

Portanto, a partir das análises dos gráficos apresentados, observa-se que nas interações relacionadas à tarefa T1 (Fórum de discussão) e T4 (troca de mensagens), 
VI Congresso Brasileiro de Informática na Educação (CBIE 2017)

Anais do XXIII Workshop de Informática na Escola (WIE 2017)

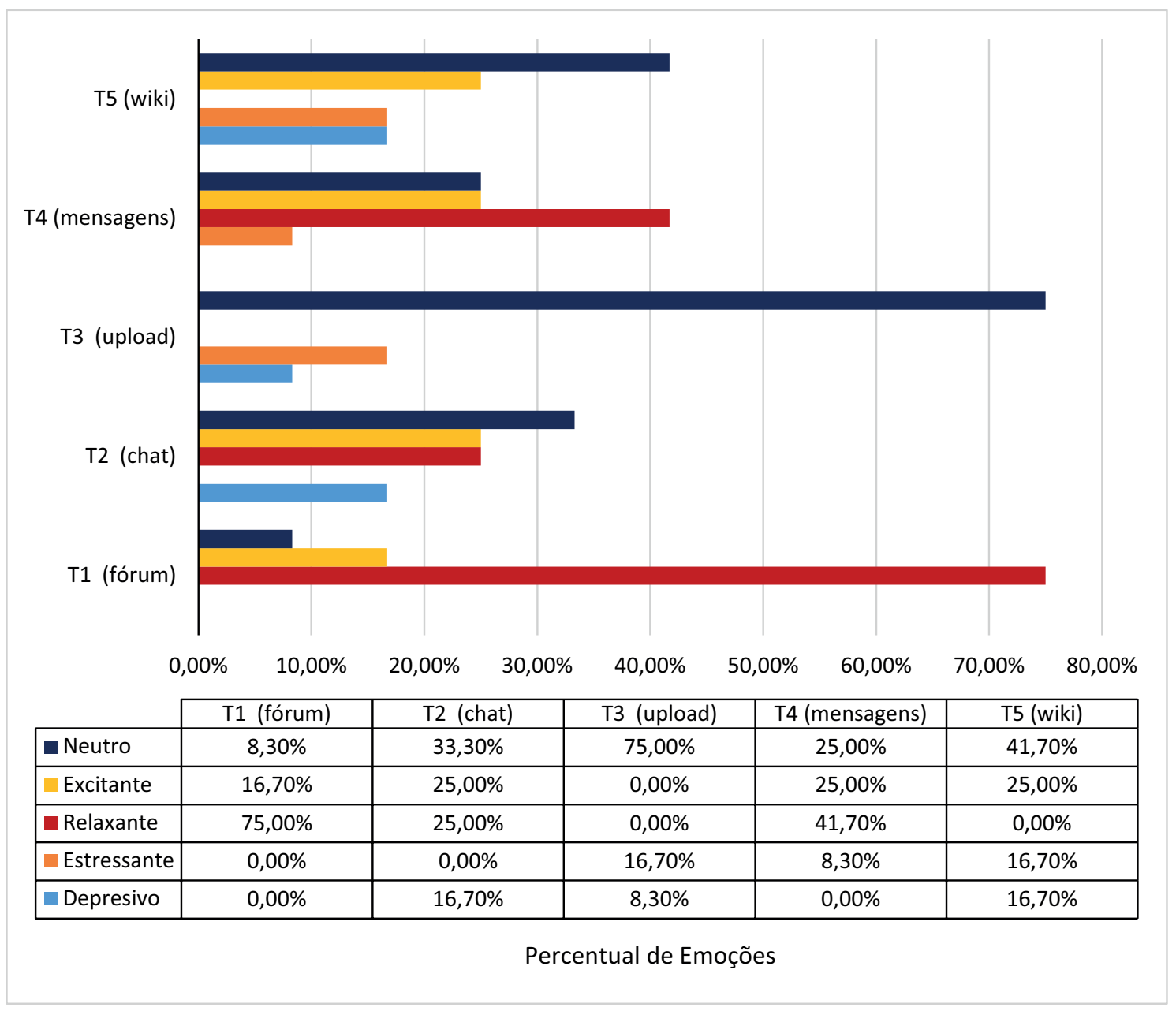

Figura 3. Percentual de respostas identificadas por emoções depressivas, estressantes, relaxantes e excitantes nas tarefas T1, T2, T3, T4 e T5.

houve um nível elevado de emoções relaxantes em relação aos demais atributos emocionais de UX. Já a tarefa T3 (uploads das atividades) apresentou um nível elevado de emoções depressivas e estressantes em relação às demais emoções. Observou-se também, que nesta tarefa, houve um nível elevado de emoções neutras. Assim, percebeu-se que as tarefas que propuseram a interação com os fóruns (T1) e trocas de mensagens no AVA do curso EaD, conseguiram um melhor impacto emocional em relação às tarefas que propuseram a realização de interação com chat da disciplina (T2), realização de uploads de atividades proposta pela disciplina (T3) e a realização de consultas ao Wiki (T5).

Estes resultados demonstram a importância da usabilidade dos ambientes virtuais de aprendizagem AVA's no processo ensino-aprendizagem dos alunos. A aplicação do método Affect Grid destacou fatores emocionais que permitiram conhecer como os estudantes interagem com as tarefas propostas, bem como seu nível de satisfação com as mesmas. Observa-se que, conhecendo-se previamente estas informações, caso este método seja adotado por instituições que atuam na educação a distancia, melhorias podem ser propostas nos AVA's visando diminuir a evasão nesta modalidade de ensino.

Sendo assim, aferir emoções no processo de interação dos estudantes com sua pla- 
VI Congresso Brasileiro de Informática na Educação (CBIE 2017)

Anais do XXIII Workshop de Informática na Escola (WIE 2017)

taforma de estudo permite o aperfeiçoamento e, ao mesmo tempo, a correção de problemas indesejados. O método Affect Grid torna-se relevante ainda, pois seus resultados despertam para a necessidade de investimentos em AVA's, visando aperfeiçoamento e maior qualidade. As novas tecnologias buscam enriquecer o processo ensino-aprendizagem de forma dinâmica, interativa, participativa e cooperativa, tornando-se essencial conhecer suas implicações a partir da manipulação de seus usuários.

\section{Conclusão}

Por meio das aplicações das tarefas nas interações com os Ambientes Virtuais de Aprendizagem (AVA) é possível mensurar a Experiência do Usuário (UX) sob a perspectiva de indicadores emocionais. Tal investigação identificou e classificou quantitativamente as respostas fornecidas pelo usuários logo após cada interação. Observou-se as tarefas sob os atributos de desprazer e prazer, sonolência e animação, nas interações propostas por todas as tarefas (T1, T2, T3, T4 e T5).

Diferentemente da usabilidade, a UX avalia os efeitos pragmáticos das interações durante as tarefas. Nesse trabalho, ao utilizar o instrumento Affect Grid foi possível realizar correlações entre as escalas de depressivo e estressante, relaxante e excitante mensuradas por meio das realizações das tarefas. Com isso, encontrou-se um nível elevado de emoções relaxantes nas tarefas que propuseram a interação com os fóruns (T1).

Os experimentos sugerem, portanto, que por meio da aplicação dos métodos de UX é possível extrair as emoções sentidas pelos alunos durante as interações em ambientes virtuais de aprendizagem. Tais resultados permitem que possíveis melhorias sejam feitas na usabilidade em ambientes colaborativos; contribuindo assim, de forma positiva para a qualidade da experiência do usuário no processo de ensino-aprendizagem nos cursos a distância.

\section{Referências}

Alves, T. M. F., Menezes, A. H. N., and Vasconcelo, F. M. d. B. P. (2015). Crescimento da educação a distância e seus desafios: Uma revisão bibliográfica. Revista de Educação do Vale do São Francisco-REVASF, 4(6):63-74.

Ferreira, V. S. and Elia, M. F. (2013). Uma modelagem conceitual para apoiar a identificação das causas da evasão escolar em ead. In Anais do Workshop de Informática na Escola, volume 1.

Frozza, R., da Silva, A. K., Lux, B., da Cruz, M. E. K., and Borin, M. (2009). Dóris 3d: Agente pedagógico baseado em emoçoes. XX Simpósio Brasileiro de Informática na Educação (SBIE).

Hartson, R. and Pyla, P. S. (2012). The UX Book: Process and guidelines for ensuring a quality user experience. Elsevier.

Likert, R. (1932). A technique for the measurement of attitudes. Archives of psychology.

Longhi, M. T., Behar, P. A., Bercht, M., and Simonato, G. (2010). Os fatores motivacionais e os estados de ânimo em ambientes virtuais de aprendizagem. In Congresso Iberoamericano de Informática Educativa. Santiago do Chile, Chile, pages 551-558.

Maule, J. (2012). Killer UX Design:Create user experiences to wow your visitors. SitePoint, 1 edition edition. 
VI Congresso Brasileiro de Informática na Educação (CBIE 2017)

Anais do XXIII Workshop de Informática na Escola (WIE 2017)

Nielsen, J. (1994). Usability engineering. Elsevier.

Nogueira, T. C., Ferreira, D. J., Carvalho, S. T., and Berreta, L. O. (2017). Evaluating responsive web design's impact on blind users. IEEE MultiMedia, 24(2):86-95.

Pereira, A. T. C., Schmitt, V., and Dias, M. (2007). Ambientes virtuais de aprendizagem: em diferentes contextos. Rio de Janeiro: Ciência Moderna Ltda, pages 2-22.

Ramos, J. L. C., Rodrigues, R. L., Silva, J. C. S., and Gomes, A. S. (2014). Analisando fatores que afetam o desempenho de estudantes iniciantes em um curso a distância. In Anais do Simpósio Brasileiro de Informática na Educação, volume 25, pages 99-108.

Rezende, F. G. C., Nunes, M. M., Brancher, J. D., and de Sordi Junior, F. (2013). Jogo eletrônico e sua influência nas emoções do usuário: Uma análise sobre como os jogos podem estimular emoções relacionadas à aprendizagem. In Anais do Simpósio Brasileiro de Informática na Educação, volume 24.

Rigo, S. J., Alves, I. M., Gazola, O., Belau, F., Barbosa, J. L., and Costa, C. (2013). Abordagem linguística para identificação da dimensão afetiva expressa em textos de ambientes virtuais de aprendizagem-um léxico da emoção. In Anais do Simpósio Brasileiro de Informática na Educação, volume 24.

Russell, J. A., Weiss, A., and Mendelsohn, G. A. (1989). Affect grid: a single-item scale of pleasure and arousal. Journal of personality and social psychology, 57(3):493.

Santos, F. D. and Bercht, M. (2014). Análise da disposição afetiva do aluno em um avea: descoberta do desânimo. In Anais do Simpósio Brasileiro de Informática na Educação, volume 25, pages 119-123.

Semesp (2016). Mapa do ensino superior no brasil. pages 140-145. Disponível em: http://www.semesp.org.br/site/pesquisas/mapa-do-ensino-superior/. Acesso em $22 \mathrm{de}$ Ago. 2017.

Sutcliffe, A. and Hart, J. (2013). Some reflections on evaluating users' experience. which is commonly known as TwinTide (Towards the Integration of Trans-sectorial IT Design, page 67 . 